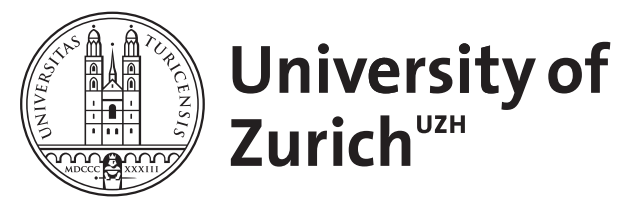

\title{
Liposome formulations of hydrophobic drugs
}

\author{
Schwendener, Reto A ; Schott, Herbert
}

\begin{abstract}
Here we report methods of preparation for liposome formulations containing lipophilic drugs. In contrast to the encapsulation of water soluble compounds into the entrapped aqueous volume of a liposome, drugs with lipophilic properties are incorporated into the phospholipid bilayer membrane. Water-soluble molecules, for example cytotoxic or antiviral nucleosides can be transformed into lipophilic compounds by attachment of long alkyl chains, allowing their stable incorporation into liposome membranes and taking advantage of the high loading capacity lipid bilayers provide for lipophilic molecules. We created a new class of cytotoxic drugs by chemical transformation of the hydrophilic drugs cytosinearabinoside (ara-C), 5-fluoro-deoxyuridine (5-FdU), and ethinylcytidine (ETC) into lipophilic compounds and their formulation in liposomes. The concept of chemical modification of water-soluble molecules by attachment of long alkyl chains and their stable incorporation into liposome bilayer membranes represent a very promising method for the development of new drugs not only for the treatment of tumors or infections but also for many other diseases.
\end{abstract}

DOI: https://doi.org/10.1007/978-1-4939-6591-5_6

Posted at the Zurich Open Repository and Archive, University of Zurich ZORA URL: https://doi.org/10.5167/uzh-134100

Book Section

Accepted Version

Originally published at:

Schwendener, Reto A; Schott, Herbert (2017). Liposome formulations of hydrophobic drugs. In: D'Souza, Gerard G M. Liposomes, Methods and Protocols, Second Edition. New York, NY, USA: Springer, 73-82. DOI: https://doi.org/10.1007/978-1-4939-6591-5_6 


\title{
Liposome Formulations of Hydrophobic Drugs
}

Reto A. Schwendener 1,*

Email rschwendener@imcr.uzh.ch

Herbert Schott 2

1 Laboratory of Liposome Research, Institute of Molecular Cancer Research, University of

Zürich, Winterthurerstrasse 190, 8057 Zurich, Switzerland

2 Institute of Organic Chemistry, Eberhard-Karls University, Auf der Morgenstelle

18, 72076 Tübingen, Germany

\section{Abstract}

Here we report methods of preparation for liposome formulations containing lipophilic drugs. In contrast to the encapsulation of water soluble compounds into the entrapped aqueous volume of a liposome, drugs with lipophilic properties are incorporated into the phospholipid bilayer membrane. Water-soluble molecules, for example cytotoxic or antiviral nucleosides can be transformed into lipophilic compounds by attachment of long alkyl chains, allowing their stable incorporation into liposome membranes and taking advantage of the high loading capacity lipid bilayers provide for lipophilic molecules. We created a new class of cytotoxic drugs by chemical transformation of the hydrophilic drugs cytosine-arabinoside (ara-C), 5-fluoro-deoxyuridine (5-FdU), and ethinylcytidine (ETC) into lipophilic compounds and their formulation in liposomes.

The concept of chemical modification of water-soluble molecules by attachment of long alkyl chains and their stable incorporation into liposome bilayer membranes represent a very promising method for the development of new drugs not only for the treatment of tumors or infections but also for many other diseases.

\section{Keywords}

\author{
Liposomes \\ Lipophilic drugs \\ Lipophilic ara-C drugs \\ NOAC \\ Duplex drugs
}

\section{Introduction}

Liposomes are predominantly used as carriers for hydrophilic molecules that are encapsulated within the aqueous inner volume which is confined by the lipid bilayer. These molecules generally do not interact with the lipid moiety of the vesicle. Long circulating liposomes modified with poly(ethylene glycol) (PEG) and other formulations carrying encapsulated cytotoxic drugs such as doxorubicin, paclitaxel, vincristine, lurtotecan, and others are clinically approved chemotherapeutic liposome formulations [1-5].

In contrast, many lipophilic drugs or prodrugs can only be applied therapeutically by use of potentially toxic solubilizing agents such as detergents or polymers or by development of complex pharmaceutical formulations [6-8]. Therefore, in view of such potential disadvantages, many hydrophobic drugs are not further developed into clinically used medicines. Such technical drawbacks can be resolved by incorporation of lipophilic drugs into the bilayer matrix of phospholipid liposomes. We and others chose the approach of the chemical transformation of water-soluble molecules of known cytotoxic properties into lipophilic drugs 
or prodrugs. Some recent examples of modifications of antitumor drugs and their formulation in liposomes are gemcitabine, paclitaxel, methotrexate, 5-iodo-2'-deoxyuridine, and cytosine arabinoside (ara-C) [9-15].

We selected ara-C as a compound of well known cytotoxic properties and transformed the nucleoside into lipophilic derivatives. Due to the insolubility of the resulting compounds we developed formulations in which the lipophilic moieties of the molecules serve as anchor for a stable incorporation into the lipid bilayer membranes of small unilamellar liposomes, taking advantage of the high loading capacity of the phospholipid bilayers. To introduce lipophilic anchors ara-C was modified with long acyl and alkyl chains, preferably of similar chain lengths as the phospholipids, allowing optimal alignment within the lipid bilayer matrix. Out of a series of $\mathrm{N}^{4}$-alkyl derivatives of ara-C, the most effective compound, $\mathrm{N}^{4}$-octadecyl-ara-C (NOAC) was extensively studied by us (Fig. 1) [16-18]. In contrast to ara-C, NOAC is a highly lipophilic drug with an extreme resistance towards deamination. Liposome formulations of NOAC showed excellent antitumor activities after oral and parenteral therapy in several tumor models. From a large number of studies we conclude that the mechanisms of action of the $\mathrm{N}^{4}$-alkyl-ara-C derivatives are distinct from ara-C and that such lipophilic derivatives represent a new class of cytotoxic nucleoside drugs [19-22]. Most hydrophobic drugs interact with lipoproteins which are the major transport vehicles for lipids and cholesterol throughout the aqueous environment of the blood and lymph circulatory systems [23, 24]. We could show that liposome-incorporated NOAC is transferred to lipoproteins, mainly to the low- and high-density lipoproteins (LDL and HDL), respectively [25-27]. Thus, the strong affinity of NOAC to lipoproteins, and of lipophilic drugs in general, might be exploited for an enhanced drug uptake in tumor cells that express high numbers of LDL receptor molecules.

Fig. 1

Chemical structures of the hydrophilic parent compound 1- $\beta-\mathrm{D}-$ arabinofuranosylcytosine (ara-C, mol. wt. 243.2) and its lipophilic $\mathrm{N}^{4}$-alkyl derivative $\mathrm{N}^{4}$-octadecyl-1- $\beta$-D-arabinofuranosylcytosine (NOAC, mol. wt. 495.7)

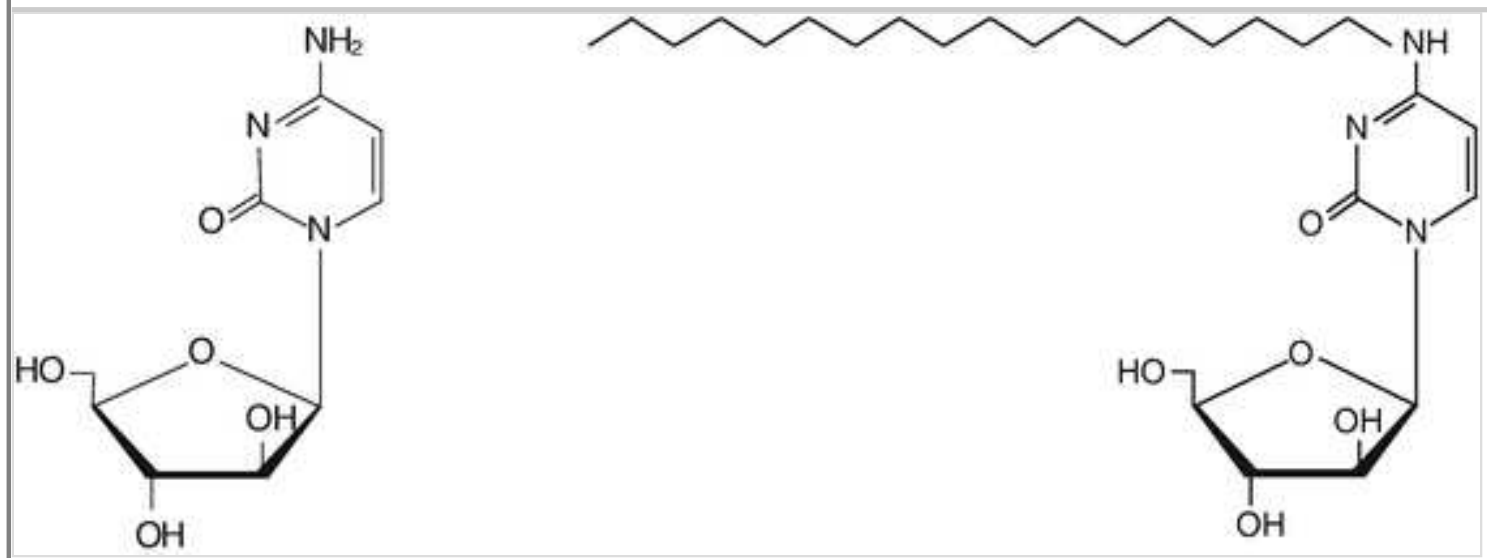

Recently, we further modified NOAC by the synthesis of new duplex drugs by combination of the clinically used cancer drugs ara-C, 5-fluorodeoxyuridine (5-FdU) and the highly active new compound ethynylcytidine (1-(3-C-ethynyl- $\beta$-D-ribopento-furanosyl)-cytosine, ETC) with NOAC, yielding the heterodinucleoside phosphates arabinocytidylyl- ${ }^{4}$-octadecyl-1- $\beta$-D-arabinofuranosyl-cytosine (ara-C-NOAC), 2'-deoxy-5fluorouridylyl-N ${ }^{4}$-octadecyl-1- $\beta$-D-arabinofuranosylcytosine (5-FdU-NOAC), and ETC-NOAC (3'-Cethynylcytidylyl-(5' $\left.\rightarrow 5^{\prime}\right)-\mathrm{N}^{4}$-octadecyl-1- $\beta$-D-arabinofuranosylcytosine) as shown in Fig. 2 [28-32]. The cytotoxic activity of such duplex drugs is expected to be more effective as compared to the monomeric nucleosides. Due to the combination of the effects of both active molecules that can be released in the cells as monomers or as the corresponding mono-phosphates it can be anticipated that mono-phosphorylated nucleosides are directly formed in the cytoplasm after enzymatic cleavage of the duplex drugs. Thus, monophosphorylated molecules would not have to pass the first phosphorylation step, which is known to be rate limiting.

Fig. 2 
Chemical structures of the $5^{\prime} \rightarrow 5^{\prime}$ phosphodiester duplex drugs ara-C-NOAC (arabinocytidylyl- $\left(5^{\prime} \rightarrow 5^{\prime}\right)$ $\mathrm{N}^{4}$-octadecyl-1- $\beta$-D-arabinofuranosylcytosine, mol. wt. 801 g/mol), 5-FdU-NOAC (2'-deoxy-5fluorouridylyl- $\left(5^{\prime} \rightarrow 5^{\prime}\right)$ - $\mathrm{N}^{4}$-octadecyl-1- $\beta$-D-arabinofuranosylcytosine, mol. wt. $\left.804 \mathrm{~g} / \mathrm{mol}\right)$ and ETCNOAC (3'-C-ethynylcytidylyl- $\left(5^{\prime} \rightarrow 5^{\prime}\right)-\mathrm{N}^{4}$-octadecyl-1- $\beta$-D-arabinofuranosylcytosine, mol. wt. 825 $\mathrm{g} / \mathrm{mol})$

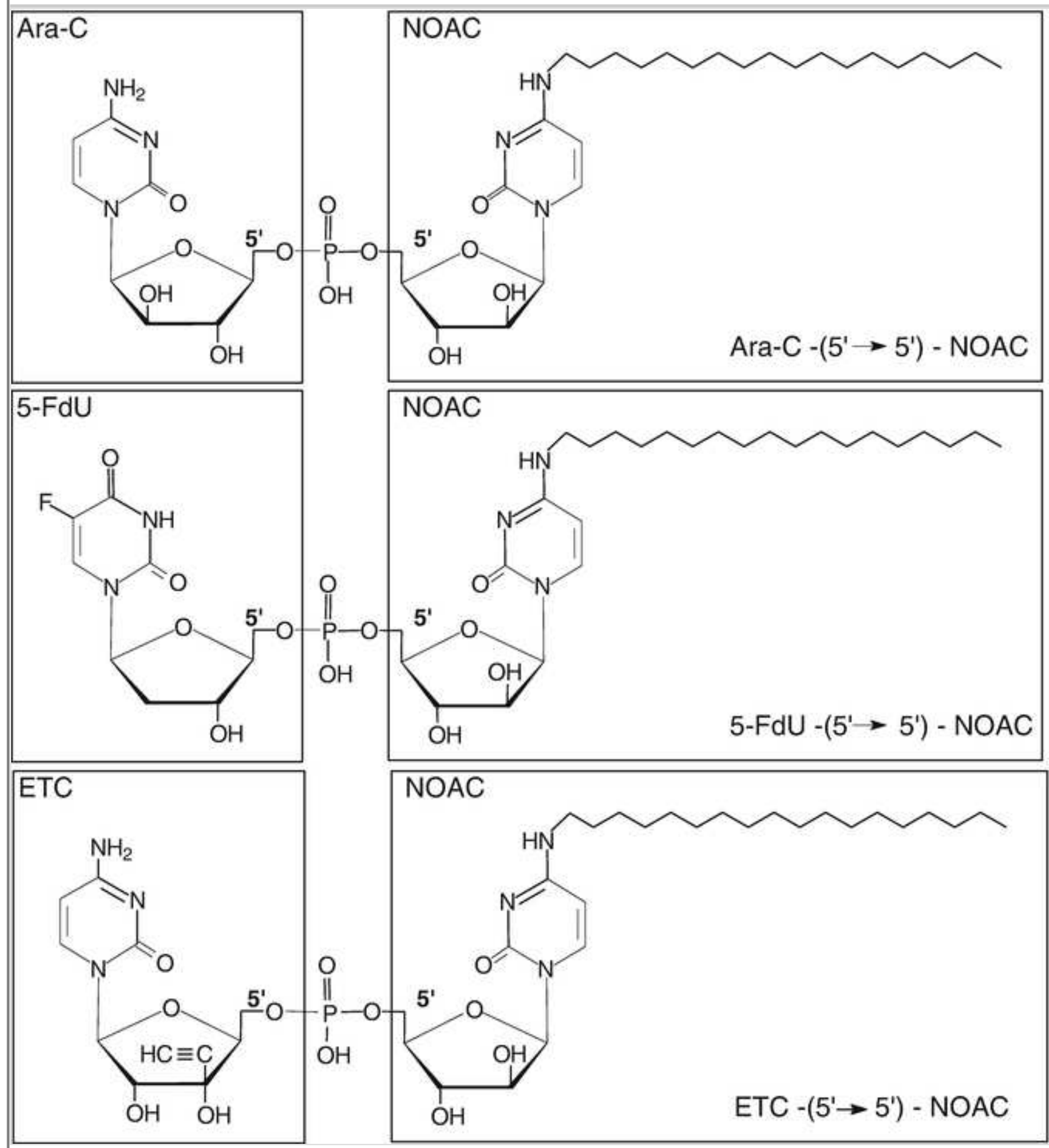

In previous studies performed with similar heterodinucleoside phosphate dimers composed of the antivirally active nucleosides azidothymidine, dideoxycytidine, and dideoxyinosine and formulated in liposomes we found significantly different pharmacokinetic properties and superior antiviral effects in comparison to the parent hydrophilic nucleosides [33, 34]. Thus, the chemical modification of cytotoxic nucleosides and their formulation in liposomes render these new hetero-dinucleoside compounds interesting candidates for further developments. 
Here, we present methods of preparation of liposomes as carriers for lipophilic nucleosides and heterodinucleoside drugs. We do not describe in detail the methods used to evaluate the cytotoxic properties of the lipophilic drug formulations. For comprehensive information we refer to our publications and to the related literature.

\section{Materials}

\subsection{Liposome Preparation (Extrusion Method)}

1. Soy phosphatidylcholine (SPC) (L. Meyer GmbH, Hamburg, Germany), store at $-20{ }^{\circ} \mathrm{C}$, prepare a stock solution for example of $20-100 \mathrm{mg} / \mathrm{ml}$ by dissolving SPC in methanol-methylene chloride $(1: 1, \mathrm{v} / \mathrm{v})$.

2. Cholesterol (Fluka, Buchs, Switzerland) (see Note 1 ).

3. D,L- $\alpha$-tocopherol (Merck, Darmstadt, Germany), store at $-20{ }^{\circ} \mathrm{C}$, make a stock solution for example of $10 \mathrm{mg} / \mathrm{ml}$ by dissolving $\mathrm{D}, \mathrm{L}-\alpha$-tocopherol in methanol-methylene chloride $(1: 1, \mathrm{v} / \mathrm{v})$.

4. Phosphate buffer, $\mathrm{PB}\left(13 \mathrm{mM} \mathrm{KH}_{2} \mathrm{PO}_{4}, 54 \mathrm{mM} \mathrm{NaHPO}_{4}, \mathrm{pH} 7.4\right)$ (see Note 3 ).

5. Round bottom flasks $(20-100 \mathrm{ml})$.

6. Rotatory evaporator, e.g., Rotavap (Büchi AG, Flawil, Switzerland).

7. Lipex ${ }^{\mathrm{TM}}$ high pression extruder [35] (Northern Lipids Inc., 8855 Northbrook Court, Burnaby, BC, Canada, Website: www.northernlipids.com ).

8. Nuclepore membranes of defined pore sizes: 400, 200, $100 \mathrm{~nm}$ (Sterlitech Corp., Kent, WA, USA or Sterico AG, Wangen, Switzerland).

9. Sterile filters 0.45 or $0.2 \mu \mathrm{m}$ and plastic syringes, various suppliers.

\section{Methods}

The methods described below outline (1) the preparation of liposomes by filter extrusion and (2), detergent dialysis.

\subsection{Preparation of NOAC, ara-C-NOAC, 5-FdU-NOAC, and ETC-NOAC Liposomes}

In the past decades a large number of methods of liposome preparation have been developed and refined. For comprehensive information we refer to corresponding chapters of this book volume and the literature. We favor the use of the two methods described below that are recommendable because of their ease, versatility, and high quality of liposomes they produce.

\subsubsection{Liposome Preparation by High Pressure Filter Extrusion}

Liposomes are prepared by sequential filter extrusion of the lipid/drug mixtures. The basic composition for the preparation of $5.0 \mathrm{ml}$ liposomes is $1.0 \mathrm{~g}$ soy phophatidylcholine (SPC, L. Meyer GmbH, Hamburg, Germany), 125 mg cholesterol (Fluka, Buchs, Switzerland) (see Note 1 ), 6 mg D,L- $\alpha$-tocopherol (Merck, Darmstadt, Germany), and the lipophilic drug at concentrations of $1-10 \mathrm{mg} / \mathrm{ml}$. The solid lipids and the lipophilic drugs (see Figs 1 and 2), either as powder or stock solutions are dissolved in methanol-methylene chloride $(1: 1, \mathrm{v} / \mathrm{v})$ in a round bottom flask (see Note 2 ). PEG-modified liposomes are obtained by addition of PEG(2000)-DPPE (28 mg/ml) to the basic lipid mixtures (see Note 3 ). After removal of the organic 
solvents by rotary evaporation $\left(40-45^{\circ} \mathrm{C}, 60 \mathrm{~min}\right)$ the dry lipid mixture is solubilized with phosphate buffer $\mathrm{PB}(67 \mathrm{mM}, \mathrm{pH} 7.4$ ) by vigorous agitation (see Note 4 ). The mixture is then subjected to repetitive extrusion through Nuclepore polycarbonate (Sterlitech Corp., Kent, WA, USA or Sterico AG, Wangen, Switzerland) filters (400, 200 and $100 \mathrm{~nm}$ pore size) using a Lipex ${ }^{\mathrm{TM}}$ Extruder (Northern Lipids, Inc.) (see

Note 5 ). Finally, the liposomes are sterilized by filtration ( 0.45 or $0.2 \mu \mathrm{m}$ sterile filters). Mean hydrodynamic diameters of vesicles (liposomes, nanospheres, nanobeads) can be determined with dynamic laser light scattering instruments, e.g., the NICOMP 380 particle sizer, Particle Sizing Systems (Sta. Barbara, CA, USA). Incorporation of the lipophilic drugs is estimated to range between 95 and $100 \%$ according to previous determinations [36] (see Note 5 ).

\subsubsection{Liposome Preparation by Dialysis}

Small unilamellar vesicles (SUV) of 50-200 nm mean size can also be prepared using detergent dialysis methods as described [37, 38]. The same lipid/drug compositions as given in Subheading 3.1.1 are used with the only difference that the detergent is also added to the organic solution. Controlled removal of detergent from mixed lipid/detergent/drug micelles yields liposomes of high size homogeneity and stability. The detergent sodium cholate (see Note 6 ) is added at a ratio of total lipids to detergent of 0.6 mol, including the lipophilic drug. The dry lipid/detergent/drug film is dispersed in PB (see Note 4 ) and left 1-2 $\mathrm{h}$ or over night at room temperature for equilibration. Detergent is removed by controlled dialysis of the mixed micelles against 3-5 1 of PB or PB-Man (volume ratio $=1-1000$ ) for $12-15 \mathrm{~h}$ at room temperature, e.g., using a Mini-Lipoprep instrument (Harvard Apparatus, Holliston, Massachusetts, Website: www.harvardapparatus.com ) (see Notes 6 and 7 ).

\subsection{Conclusions}

With the chemical transformation of water-soluble nucleosides into lipophilic compounds, followed by their incorporation into lipid bilayer membranes of liposomes, a new class of cytotoxic drug formulations is obtained that can be applied for the treatment of tumors by parenteral and oral routes. Lipophilic ara-C derivatives, particularly the extensively studied drug NOAC, and the novel duplex drugs composed of NOAC and the nucleosides ara-C, 5-FdU, and ETC represent very promising new anticancer drugs of high cytotoxic activity, ability to circumvent resistance mechanisms, and strong apoptosis inducing capability.

We conclude that the chemical modification of water-soluble molecules by attachment of long alkyl chains and their stable incorporation into the bilayer membranes of small unilamellar liposomes represent a very promising example of taking advantage of the high loading capacity lipid bilayers offer for lipophilic drugs. The combination of chemical modifications of water soluble drugs of known pharmacological activities with their formulation in liposomes represents a valuable method for the development of novel pharmaceutical preparations, not only for the treatment of tumors or infectious diseases but also for many other disorders.

\section{Notes}

1. Cholesterol (e.g., from Fluka, purum quality, $>95 \%$ ) should be recrystallized from methanol. Cholesterol of minor quality or purity should be avoided, since liposome membrane stability can be reduced.

2. Detachment of the lipid mixtures from the glass walls of the round bottom flasks can be accelerated by addition of small glass beads ( $2-3 \mathrm{~mm}$ diameter) and vigorous shaking. Preferably, the glass beads are added to the organic lipid solution before evaporation of the solvents. This will facilitate detachment and dispersion of the lipid film.

3. Other lipid compositions with synthetic lipids, hydrogenated SPC (HSPC), and PEG-modified phospholipids are often used, especially for liposome formulations intended for parenteral applications use (long circulating or "stealth" liposomes) [39]. Several analytical methods to follow loss of lipids during the preparation steps are available. Radioactively labeled lipids $\left({ }^{3} \mathrm{H}-\mathrm{DPPC},{ }^{14} \mathrm{C}\right.$ DPPC) or cholesterol $\left({ }^{3} \mathrm{H}\right.$-cholesterol) or ${ }^{3} \mathrm{H}$-cholesteryl hexadecyl ether (NEN Life Science 
Products, Boston, MA, USA) or lipophilic fluorescence dyes (e.g., lipophilic BODIPY derivatives, Molecular Probes) are added at appropriate amounts to the initial lipid mixtures.

4. If the liposome preparations are intended to be stored for longer time periods they may be frozen or lyophilized, provided that they are prepared in a phosphate buffer that contains a cryoprotectant. We use an iso-osmolar phosphate-mannitol buffer of the following composition: $20 \mathrm{mM}$ phosphate buffer $\left(0.53 \mathrm{~g} / 1 \mathrm{KH}_{2} \mathrm{PO}_{4}\right.$ plus $\left.2.87 \mathrm{~g} / 1 \mathrm{Na}_{2} \mathrm{HPO}_{4} \cdot 2 \mathrm{H}_{2} \mathrm{O}\right)$ plus $230 \mathrm{mM}$ mannitol (42.0 g/l mannitol).

5. The concentration of the lipophilic drugs NOAC, ara-C-NOAC, 5-FdU-NOAC, or ETC-NOAC in the liposomes can be varied from $1 \mathrm{mg} / \mathrm{ml}$ to about $10 \mathrm{mg} / \mathrm{ml}$, depending on the concentration required for biological activity (e.g., based on corresponding $\mathrm{IC}_{50}$-values), the phospholipid concentration, the lipid composition and the method of liposome preparation. The concentrations of incorporated drugs can be determined by reverse phase HPLC [36].

6. The preparation of liposomes from mixed detergent/lipid micelles can also be done with other detergents, such as n-alkyl-glucosides $(n=6-9)$, octyl-thioglucoside or $N$-octanoyl- $N$ methylglucamine (MEGA-8, Fluka). Interestingly, the choice of detergent influences the size of the resulting liposomes. Thus, liposomes prepared from n-octyl-glucoside-phospholipid-cholesterol mixed micelles have an average size of $180 \mathrm{~nm}$, whereas those made with n-hexyl-glucoside are 60 $\mathrm{nm}$ in diameter [37]. Detergent removal by conventional dialysis using semipermeable dialysis tubes (e.g., Spectrapor, mol. wt. cutoff 12-14,000 Da) is not recommended because, due to a concentration gradient which is formed within the dialysis tube, heterogenous and unstable liposomes will be produced.

7. When synthetic lipids are used, detergent removal has to be performed above the corresponding transition temperature $T_{\mathrm{c}}$ of the lipid. Hence, when for example dipalmitoylphosphatidyl choline (DPPC) is used as main liposome forming lipid, a temperature above its $T_{\mathrm{c}}$ of $41{ }^{\circ} \mathrm{C}$ has to be chosen. Additional membrane forming components (cholesterol, lipophilic drugs, etc.) depress the $T$ c by several degrees.

\section{References}

1. Hofheinz RD, Gnad-Vogt SU, Beyer U, Hochhaus A (2005) Liposomal encapsulated anti-cancer drugs. Anticancer Drugs 16:691-707

2. Perez-Lopez ME, Curiel T, Gomez JG, Jorge M (2007) Role of pegylated liposomal doxorubicin (Caelyx) in the treatment of relapsing ovarian cancer. Anticancer Drugs 18:611-617

3. Porter CA, Rifkin RM (2007) Clinical benefits and economic analysis of pegylated liposomal doxorubicin/vincristine/dexamethasone versus doxorubicin/vincristine/dexamethasone in patients with newly diagnosed multiple myeloma. Clin Lymphoma Myeloma S4:S150-S155

4. Thomas DA, Sarris AH, Cortes J, Faderl S, O'Brien S, Giles FJ, Garcia-Manero G, Rodriguez MA, Cabanillas F, Kantarjian H (2006) Phase II study of sphingosomal vincristine in patients with recurrent or refractory adult acute lymphocytic leukemia. Cancer 106:120-127

5. Hennenfent KL, Govindan R (2006) Novel formulations of taxanes: a review. Old wine in a new bottle? Ann Oncol 17:735-749

6. Strickley RG (2004) Solubilizing excipients in oral and injectable formulations. Pharm Res 21:201-230

7. Fahr A, Liu X (2007) Drug delivery strategies for poorly water-soluble drugs. Expert Opin Drug Deliv 4:403-416 
8. ten Tije AJ, Verweij J, Loos WJ, Sparreboom A (2003) Pharmacological effects of formulation vehicles: implications for cancer chemotherapy. Clin Pharmacokinet 42:665-685

9. Brusa P, Immordino ML, Rocco F, Cattel L (2007) Antitumor activity and pharmacokinetics of liposomes containing lipophilic gemcitabine prodrugs. Anticancer Res 27:195-199

10. Bergman AM, Kuiper CM, Noordhuis P, Smid K, Voorn DA, Comijn EM, Myhren F, Sandvold ML, Hendriks HR, Fodstad O, Breistol K, Peters GJ (2004) Antiproliferative activity and mechanism of action of fatty acid derivatives of gemcitabine in leukemia and solid tumor cell lines and in human xenografts. Nucleosides Nucleotides Nucleic Acids 23:1329-1333

11. Stevens PJ, Sekido M, Lee RJ (2004) A folate receptor-targeted lipid nanoparticle formulation for a lipophilic paclitaxel prodrug. Pharm Res 21:2153-2157

12. Pignatello R, Puleo A, Puglisi G, Vicari L, Messina A (2003) Effect of liposomal delivery on in vitro antitumor activity of lipophilic conjugates of methotrexate with lipoamino acids. Drug Deliv 10:95-100

13. Zerouga M, Stillwell W, Jenski LJ (2002) Synthesis of a novel phosphatidylcholine conjugated to docosahexaenoic acid and methotrexate that inhibits cell proliferation. Anticancer Drugs 13:301-311

14. Harrington KJ, Syrigos KN, Uster PS, Zetter A, Lewanski CR, Gullick WJ, Vile RG, Stewart JS (2004) Targeted radiosensitisation by pegylated liposome-encapsulated 3', 5'-O-dipalmitoyl 5-iodo-2'deoxyuridine in a head and neck cancer xenograft model. Br J Cancer 91:366-373

15. Hamada A, Kawaguchi T, Nakano M (2002) Clinical pharmacokinetics of cytarabine formulations. Clin Pharmacokinet 41:705-718

16. Rubas W, Supersaxo A, Weder HG, Hartmann HR, Hengartner H, Schott H, Schwendener RA (1986) Treatment of murine L1210 leukemia and melanoma B16 with lipophilic cytosine arabinoside prodrugs incorporated into unilamellar liposomes. Int J Cancer 37:149-154

17. Schwendener RA, Schott H (1992) Treatment of L1210 murine leukemia with liposome - incorporated $\mathrm{N}^{4}$-hexadecyl-1- $\beta$-D-arabino-furanosyl-cytosine. Int J Cancer 51:466-469

18. Schwendener RA, Schott H (2005) Lipophilic arabinofuranosyl cytosine derivatives in liposomes. Meth Enzymol 391:58-70

19. Horber DH, Schott H, Schwendener RA (1995) Cellular pharmacology of a liposomal preparation of $\mathrm{N}^{4}$ hexadecyl-1- $\beta$-D-arabino-furanosylcytosine, a lipophilic derivative of 1- $\beta$-D-arabinofuranosylcytosine. $\mathrm{Br}$ J Cancer 71:957-962

20. Horber DH, von Ballmoos P, Schott H, Schwendener RA (1995) Cell cycle dependent cytotoxicity and induction of apoptosis by $\mathrm{N}^{4}$-hexadecyl-1- $\beta$-D-arabinofuranosylcytosine, a new lipophilic derivative of 1$\beta$-D-arabino-furanosylcytosine. Br J Cancer 72:1067-1073

21. Horber DH, Schott H, Schwendener RA (1995) Cellular pharmacology of $N^{4}$-hexadecyl-1- $\beta$-Darabinofuranosylcytosine (NHAC) in the human leukemic cell lines K-562 and U-937. Cancer Chemother Pharmacol 36:483-492

22. Schwendener RA, Friedl K, Depenbrock H, Schott H, Hanauske AR (2001) In vitro activity of liposomal $\mathrm{N}^{4}$ octadecyl-1- $\beta$-D-arabino-furanosylcytosine (NOAC), a new lipophilic derivative of $1-\beta$-D-arabinofuranocylcytosine on biopsized clonogenic human tumor cells and hematopoietic precursor cells. Invest New Drugs 19:203-210

23. Wasan KM, Brocks DR, Lee SD, Sachs-Barrable K, Thornton SJ (2008) Impact of lipoproteins on the 
biological activity and disposition of hydrophobic drugs: implications for drug discovery. Nat Rev Drug Discov 7:84-99

24. Rensen PC, de Vrueh RL, Kuiper J, Bijsterbosch MK, Biessen EA, van Berkel TJ (2001) Recombinant lipoproteins: lipoprotein-like lipid particles for drug targeting. Adv Drug Deliv Rev 47:251-276

25. Koller-Lucae SKM, Schott H, Schwendener RA (1997) Pharmacokinetic properties in mice and interactions with human blood in vitro of liposomal $\mathrm{N}^{4}$-octadecyl-1- $\beta$-D-arabinofuranosylcytosine (NOAC), a new anticancer drug. J Pharmacol Exp Thera 282:1572-1580

26. Koller-Lucae SKM, Suter MJ, Rentsch KM, Schott H, Schwendener RA (1999) Metabolism of the new liposomal anticancer drug $\mathrm{N}^{4}$-octadecyl-1- $\beta$-D-arabinofuranosylcytosine (NOAC) in mice. Drug Metab Dispos 27:342-350

27. Koller-Lucae SM, Schott H, Schwendener RA (1999) Low density lipoprotein and liposome mediated uptake and cytotoxic effect of $\mathrm{N}^{4}$-octadecyl-1- $\beta$-D-arabinofuranosylcytosine (NOAC) in Daudi lymphoma cells. Br J Cancer 80:1542-1549

28. Horber DH, Cattaneo-Pangrazzi RM, von Ballmoos P, Schott H, Ludwig PS, Eriksson S, Fichtner I, Schwendener RA (2000) Cytotoxicity, cell cycle perturbations and apoptosis in human tumor cells by lipophilic $\mathrm{N}^{4}$-alkyl-1- $\beta$-D-arabinofuranosylcytosine derivatives and the new heteronucleoside phosphate dimer arabinocytidylyl- $\left(5^{\prime} \rightarrow 5^{\prime}\right)-\mathrm{N}^{4}$-octadecyl-1- $\beta$-D-ara-C. J Cancer Res Clin Oncol 126:311-319

29. Cattaneo-Pangrazzi RM, Schott H, Wunderli-Allenspach H, Derighetti M, Schwendener RA (2000) Induction of cell cycle-dependent cytotoxicity and apoptosis by new heterodinucleoside phosphate dimers of 5-fluorodeoxyuridine in PC-3 human prostate cancer cells. Biochem Pharmacol 60:1887-1896

30. Cattaneo-Pangrazzi RM, Schott H, Schwendener RA (2000) The novel heterodinucleoside dimer 5-FdUNOAC is a potent cytotoxic drug and a p53-independent inducer of apoptosis in the androgenindependent human prostate cancer cell lines PC-3 and DU-145. Prostate 45:8-18

31. Marty C, Ballmer-Hofer K, Neri D, Klemenz R, Schott H, Schwendener RA (2002) Cytotoxic targeting of F9 teratocarcinoma tumours with anti-ED-B fibronectin $\mathrm{scFv}$ antibody modified liposomes. Br J Cancer 87:106-112

32. Takatori S, Kanda H, Takenaka K, Wataya Y, Matsuda A, Fukushima M, Shimamoto Y, Tanaka M, Sasaki T (1999) Antitumor mechanisms and metabolism of the novel antitumor nucleoside analogues, 1(3-C-ethynyl- $\beta$-D-ribo-pentofuranosyl)cytosine and 1-(3-C-ethynyl- $\beta$-D-ribo-pentofuranosyl)uracil. Cancer Chemother Pharmacol 44:97-104

33. Schwendener RA, Gowland P, Horber DH, Zahner R, Schertler A, Schott H (1994) New lipophilic acyl/alkyl dinucleoside phosphates as derivatives of 3'-azido-3'-deoxythymidine: inhibition of HIV-1 replication in vitro and antiviral activity against Rauscher leukemia virus infected mice with delayed treatment regimens. Antiviral Res 24:79-93

34. Peghini PA, Zahner R, Kuster H, Schott H, Schwendener RA (1998) In vitro inhibition of hepatitis B virus replication and pharmacokinetic properties of new lipophilic dinucleoside phosphate derivatives. Antivir Chem Chemother 9:117-126

35. Mayer LD, Hope MJ, Cullis PR (1986) Vesicles of variable sizes produced by a rapid extrusion procedure. Biochim Biophys Acta 858:161-168

36. Rentsch KM, Schwendener RA, Schott H, Hänseler E (1997) Pharmacokinetics of $N^{4}$-octadecyl-1- $\beta$-Darabinofuranosylcytosine (NOAC) in plasma and whole blood after intravenous and oral application in mice. J Pharm Pharmacol 49:1076-1081 
37. Schwendener RA, Asanger M, Weder HG (1981) The preparation of large bilayer liposomes: controlled removal of n-alkyl-glucoside detergents from lipid/detergent micelles. Biochem Biophys Res Commun 100:1055-1062

38. Schwendener RA (1986) The preparation of large volumes of homogeneous, sterile liposomes containing various lipophilic cytostatic drugs by the use of a capillary dialyzer. Cancer Drug Deliv 3:123-129

39. Allen TM (1994) Long-circulating (sterically stabilized) liposomes for targeted drug delivery. Trends Pharmacol Sci 15:215-220 